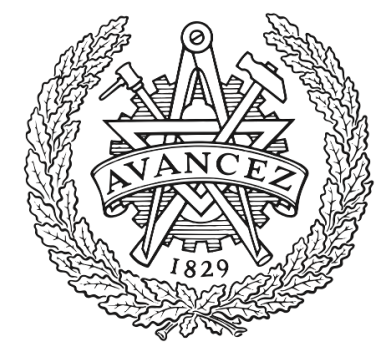

CHALMERS

UNIVERSITY OF TECHNOLOGY

\title{
Serum neopterin levels in relation to mild and severe COVID-19
}

Downloaded from: https://research.chalmers.se, 2023-04-26 10:53 UTC

Citation for the original published paper (version of record):

Robertson, J., Gostner, J., Nilsson, S. et al (2020). Serum neopterin levels in relation to mild and severe COVID-19. BMC Infectious Diseases, 20(1). http://dx.doi.org/10.1186/s12879-020-05671-7

N.B. When citing this work, cite the original published paper. 


\title{
Serum neopterin levels in relation to mild and severe COVID-19
}

Josefina Robertson ${ }^{1,2^{*}}$ (D) Johanna M. Gostner ${ }^{3}$, Staffan Nilsson ${ }^{4}$, Lars-Magnus Andersson ${ }^{1,2}$, Dietmar Fuchs ${ }^{5}$ and Magnus Gisslen ${ }^{1,2}$

\begin{abstract}
Background: The COVID-19 pandemic, caused by the coronavirus SARS-CoV-2, is rapidly spreading worldwide. There is limited information about prognostic markers that could help clinicians to identify COVID-19 patients with a poor prognosis. Serum levels of the immune activation marker neopterin has shown to be of prognostic value in patients with SARS. The aim of this study was to investigate whether serum neopterin is associated with the severity of COVID-19.

Methods: We included 34 patients with confirmed COVID-19 between March 3 and March 30, 2020. Fifteen patients had mild disease and did not require hospitalization, whereas 19 patients developed severe COVID-19 requiring intensive care. Concentrations of serum neopterin, tryptophan, and kynurenine were measured at and repeatedly after inclusion.

Results: We found a more than two-fold higher mean concentration of neopterin in severely ill patients (mean value $42.0 \mathrm{nmol} / \mathrm{L}$ (SD 18.2)) compared to patients with mild symptoms (16.9 nmol/L (SD 11.0)). All of the severe cases had elevated neopterin concentrations $(>9.1 \mathrm{nmol} / \mathrm{L}$ ) at the initial sampling with values ranging from 17.2 to $86.7 \mathrm{nmol} / \mathrm{L}$. In comparison, 10 of 15 patients with mild disease had neopterin levels above $9.1 \mathrm{nmol} / \mathrm{L}$, with concentrations in the range from 4.9 to $31.6 \mathrm{nmol} / \mathrm{L}$. Neopterin levels gradually decreased during the course of COVID-19, but severe cases maintained elevated levels for a longer period. Moreover, lower levels of tryptophan and higher levels of kynurenine, indicating an increased tryptophan catabolism, were seen in the group with severe cases.
\end{abstract}

Conclusions: In conclusion, we found that serum neopterin levels are associated with the severity of COVID-19. Our findings suggest that neopterin could be used as a prognostic marker, but further studies are needed to elucidate how it can be used in the clinic.

Keywords: COVID-19, Prognostic markers, Neopterin, Tryptophan, Kynurenine

\section{Introduction}

The COVID-19 pandemic, caused by the coronavirus SARS-CoV-2, is rapidly spreading worldwide [1]. Most patients have mild symptoms from the upper respiratory tract, whereas a minor but not negligible proportion suffers from a severe form of the disease, which in some cases require intensive care [2]. There is limited

\footnotetext{
* Correspondence: josefina.robertsson@vgregion.se

'Department of Infectious Diseases, Institute of Biomedicine, Sahlgrenska Academy, University of Gothenburg, Gothenburg, Sweden

${ }^{2}$ Department of Infectious Diseases, Region Västra Götaland, Sahlgrenska University Hospital, Gothenburg, Sweden

Full list of author information is available at the end of the article
}

information about prognostic markers that could help clinicians to identify COVID-19 patients with a poor prognosis. During the outbreak of the severe acute respiratory syndrome (SARS) in 2002-2003, caused by the similar coronavirus SARS-CoV, levels of the immune activation marker neopterin were found to predict the course of disease [3].

Neopterin (6-(D-erythro-1', 2', 3'-trihydroxypropyl)pterin) is a well-established immune activation marker with elevated concentrations seen in many inflammatory states including infections, autoimmune disorders, and cancer [4]. In acute viral infections such as hepatitis [5],

(c) The Author(s). 2020 Open Access This article is licensed under a Creative Commons Attribution 4.0 International License, which permits use, sharing, adaptation, distribution and reproduction in any medium or format, as long as you give appropriate credit to the original author(s) and the source, provide a link to the Creative Commons licence, and indicate if changes were made. The images or other third party material in this article are included in the article's Creative Commons licence, unless indicated otherwise in a credit line to the material. If material is not included in the article's Creative Commons licence and your intended use is not permitted by statutory regulation or exceeds the permitted use, you will need to obtain permission directly from the copyright holder. To view a copy of this licence, visit http://creativecommons.org/licenses/by/4.0/ The Creative Commons Public Domain Dedication waiver (http://creativecommons.org/publicdomain/zero/1.0/) applies to the data made available in this article, unless otherwise stated in a credit line to the data. 
Cytomegalovirus disease [6], Rubella [7], and dengue fever [8], serum neopterin levels correlate with the activity of the disease, and can be detected before antibody production [4]. The elevation of neopterin originates mainly from the increased synthesis by human monocyte-derived macrophages, whereby interferon-gamma (IFN-y) is the most central activating cytokine [9]. IFN-y also promotes the conversion of the essential amino acid L-tryptophan (TRP) to $\mathrm{N}$-formylkynurenine, which is rapidly converted into the more stable kynurenine (KYN), by induction of the antiproliferative and immunoregulatory enzyme indoleamine 2 , 3-dioxygenase (IDO). The first step of the TRP breakdown is the rate-limiting step in the TRP catabolic route along the KYN axis, and the KYN to TRP ratio can be used as a measure of the IDO enzyme activity [10]. Taken together, IFN-y mediated immune response to viral infections may lead to elevated neopterin levels, as well as increased TRP degradation and elevated KYN to TRP ratio $[4,11]$.

In patients with SARS, elevated levels of neopterin were detected already at the day of symptom onset, and rose to a maximum level at day 3 [3]. Moreover, patients with higher levels of neopterin at an early stage suffered from a severer course of disease, including higher and longer period of fever, more severe dyspnea, longer hospitalization, and more complications [3]. Considering these findings and the similarity of the coronaviruses, neopterin may be a useful prognostic marker for the course of COVID-19. The aim of this study was to investigate whether serum neopterin levels in COVID-19 patients are associated with the severity of disease.

\section{Methods}

\section{Participants}

We included 34 patients with COVID-19, who were admitted to the Department of Infectious Diseases at the Sahlgrenska University Hospital, Gothenburg, Sweden. All cases were confirmed with reverse transcriptase polymerase chain reaction (RT-PCR) from nasopharyngeal and throat aspirates. Fifteen patients had mild disease and did not require hospitalization, whereas 19 patients developed severe COVID-19 that in most cases required intensive care. Blood samples were collected between March 3 and March 30, 2020.

\section{Serum neopterin, TRP, KYN, and IFN-y measurements}

Serum neopterin concentrations were determined using enzyme-linked immunosorbent assay (ELISA) (BRAHMS $\mathrm{GmbH}$, Hennigsdorf, Germany) as described by the manufacturer's instructions. Serum samples and standards were treated with Igepal (Sigma-Aldrich, Vienna, Austria; final concentration in serum or standards was $2 \%(\mathrm{v} / \mathrm{v}))$. Sensitivity of the test was $2 \mathrm{nmol} / \mathrm{L}$ neopterin. The upper normal reference level was $9.1 \mathrm{nmol} / \mathrm{L}$ in serum [12]. Serum TRP and KYN concentrations were measured by a reverse-phase HPLC method [13], using a Varian ProStar HPLC system equipped with a solvent delivery module (model 210), an autosampler (model 400, both Varian ProStar), an UV-spectrometric detector (SPD-6A, Shimadzu), and a fluorescence detector (model 360, Varian ProStar). Varian Star Chromatography Workstation (version 6.30) software was used. To analyze serum IFN-y, we used the human IFN-gamma Quantikine ELISA (R\&D-DIF50C) according to the manufacturer's instructions.

\section{Statistical analyses}

Descriptive statistics are shown for all variables involved in the analyses, presented as means with standard deviations. For statistical analyses, continuous variables were $\log _{10}$ transformed. Student's t-test was used for group comparisons. Changes in log concentrations from first to last measure were analyzed with paired t-test. Associations were measured with Pearson correlation. To study the change in neopterin levels over time, we used a linear mixed effects model with severity (mild/severe), days since symptom onset, and age as covariates. An interaction term between severity and days since onset was included in the model. All statistical analyses were performed with the Statistical Package for the Social Sciences (SPSS) software version 25 (SPSS, Chicago, Illinois, USA) or Prism (GraphPad software version 8.0, La Jolla, California, USA). A significance level below 0.05 was considered as statistically significant.

\section{Results}

The study population comprised 34 patients with COVID-19, of which 15 displayed mild symptoms (mean age 51.3 years (SD 13.7)), and 19 developed a severe form of the disease (mean age 61.3 years (SD 11.4)). Cough, myalgia, nasal congestion, fever, and sore throat were the most common symptoms among mild cases, whereas fever, cough, dyspnea, and fatigue were most frequent in severe cases (Table 1). Among severe cases, all but four required invasive mechanical ventilation, and three were in need of continuous renal replacement therapy. Four deaths occurred in this group. Comorbidities among mild cases were rare, while a majority of severe cases had one or more comorbidities, of which hypertension and diabetes mellitus were most frequent (Table 1).

To test whether neopterin levels are associated with the severity of COVID-19, we compared serum neopterin concentrations between patients with mild and severe disease. We found a more than two-fold higher mean concentration of neopterin in severely ill patients (mean value $42.0 \mathrm{nmol} / \mathrm{L}$ (SD 18.2)) compared to patients with mild symptoms $(16.9 \mathrm{nmol} / \mathrm{L}$ (SD 11.0)) (Table 2, Figs. 1 and 2). All of the severe cases had 
Table 1 Clinical characteristics of included patients with mild and severe COVID-19

\begin{tabular}{lll}
\hline & $\begin{array}{l}\text { Mild disease } \\
(\boldsymbol{n}=\mathbf{1 5})\end{array}$ & $\begin{array}{l}\text { Severe disease } \\
(\boldsymbol{n}=\mathbf{1 9})\end{array}$ \\
\hline Gender (M/F) & $6 / 9$ & $17 / 2$ \\
Age, years (SD) & $51.3(13.7)$ & $61.3(11.4)$ \\
Comorbidity, n (\%) & $2(13)$ & $11(58)$ \\
Hypertension & $0(0)$ & $11(58)$ \\
Diabetes mellitus & $1(7)$ & $6(32)$ \\
Coronary heart disease & $1(7)$ & $3(16)$ \\
Chronic obstructive & $0(0)$ & $1(5)$ \\
pulmonary disease & & $1(5)$ \\
Asthma & $0(0)$ & $19(100)$ \\
Symptoms, n (\%) & & $18(95)$ \\
Fever & $9(60)$ & $17(89)$ \\
Cough & $14(93)$ & $12(63)$ \\
Dyspnea & $1(7)$ & $5(26)$ \\
Fatigue & $3(20)$ & $2(11)$ \\
Myalgia & $11(73)$ & $1(5)$ \\
Sore throat & $8(53)$ & $17(89)$ \\
Nasal congestion & $11(73)$ & $15(79)$ \\
Intensive care, n (\%) & $0(0)$ & $3(16)$ \\
Invasive mechanical & $0(0)$ & \\
ventilation, $\mathrm{n}(\%)$ & $0(0)$ & \\
Continuous renal \\
replacement therapy, $\mathrm{n}(\%)$ \\
Death, n (\%)
\end{tabular}

$M$ males, $F$ females

elevated neopterin concentrations $(>9.1 \mathrm{nmol} / \mathrm{L})$ at the initial sampling with values ranging from 17.2 to 86.7 $\mathrm{nmol} / \mathrm{L}$, measured at day 4-20 (mean 11.7 (SD 5.8); median 14) since onset of symptoms. In comparison, 10 of 15 patients with mild disease had neopterin levels above $9.1 \mathrm{nmol} / \mathrm{L}$ at day 2-19 (mean 10.2 (SD 9); median 9), with concentrations for the entire group in the range from 4.9 to $31.6 \mathrm{nmol} / \mathrm{L}$. These results show that patients with severe COVID-19 display higher levels of

Table 2 Concentrations of neopterin and amino acids from the study population divided into two groups based on disease severity

\begin{tabular}{llll}
\hline & $\begin{array}{l}\text { Mild }(\boldsymbol{n}=15) \\
\text { Mean (SD) }\end{array}$ & $\begin{array}{l}\text { Severe }(\boldsymbol{n}=19) \\
\text { Mean (SD) }\end{array}$ & $\boldsymbol{p}$-value \\
\hline Days since onset & $10.2(9.0)$ & $11.7(5.8)$ & \\
Neopterin, nmol/L & $16.9(11.0)$ & $42.0(18.2)$ & $p<0.0001$ \\
Tryptophan, $\mu \mathrm{mol} / \mathrm{L}$ & $56.3(11.6)$ & $31.9(13.0)$ & $p<0.0001$ \\
Kynurenine, $\mu \mathrm{mol} / \mathrm{L}$ & $2.6(0.7)$ & $4.3(1.4)$ & $p<0.0001$ \\
KYN/TRP *1000 & $47.5(15.1)$ & $163.4(117.5)$ & $p<0.0001$ \\
IFN-y, pg/mL & $6.7(5.0)$ & $21.4(20.8)$ & $p<0.001$ \\
\hline
\end{tabular}

Means and standard deviations (SD) are shown

KYN/TRP kynurenine to tryptophan ratio, IFN-y interferon-gamma neopterin than mild cases. Since renal insufficiency may affect serum neopterin levels [14], we analyzed creatinine levels in relation to neopterin in severe cases. However, no significant correlation was found $(r=0.36, p=$ 0.13). Moreover, we found no association between neopterin levels and C-reactive protein (CRP) or Blymphocytes among the severely ill (data not shown).

As a next step, we studied the trajectory of neopterin during the progression of COVID-19 by repeated measurements. We found that serum neopterin levels decreased over time, regardless of mild or severe disease $(p<0.0001$, Fig. 3$)$. At day 14 after symptom onset, the group with severe disease displayed 2.3 times higher neopterin levels than patients with mild disease. Moreover, neopterin concentrations decreased $23 \%$ per week in severe cases compared to $31 \%$ per week in mild cases. The difference in slope was, however, not significant $(p=0.23)$. Twelve of the patients with mild disease had normal levels $(<9.1 \mathrm{nmol} / \mathrm{L})$ at the last measurement. In contrast, only one patient among the severely ill returned to a normal level during the study period (Fig. 3 ). These results show that neopterin levels gradually decrease during the course of COVID-19, but that severe cases maintain elevated levels for a longer period. Collectively, our findings indicate an association between neopterin and the severity of COVID-19.

Furthermore, we investigated TRP, KYN, and IFN-y concentrations in relation to mild and severe COVID19. As seen in Fig. 1, we found lower levels of TRP and higher levels of KYN in severe cases, as compared to mild cases. This indicates that a severe form of COVID19 is associated with an increased TRP catabolism. Additionally, KYN/TRP concentrations closely correlated to neopterin ( $r=0.7, p<0.0001$, Fig. 4). Analysis of IFN-y in serum revealed significantly higher levels in severe cases compared to mild cases (Table 2), and the levels of IFN-y correlated to neopterin $(r=0.8, p<0.0001)$.

\section{Discussion}

In the present study, we investigated if serum neopterin levels in patients with COVID-19 are associated with the severity of disease. We report that higher neopterin concentrations were seen in patients who developed severe disease compared to patients with mild disease. In the group with severe COVID-19, we also found an increased metabolism of TRP, as expressed by higher KYN to TRP ratio.

The observed difference in neopterin concentrations between mild and severe COVID-19 is in agreement with previous results from SARS patients [3]. In that study, higher neopterin levels were associated with a longer fever period, as well as a severer course of disease. Moreover, a recent report on hospitalized COVID-19 patients showed that neopterin levels were significantly 

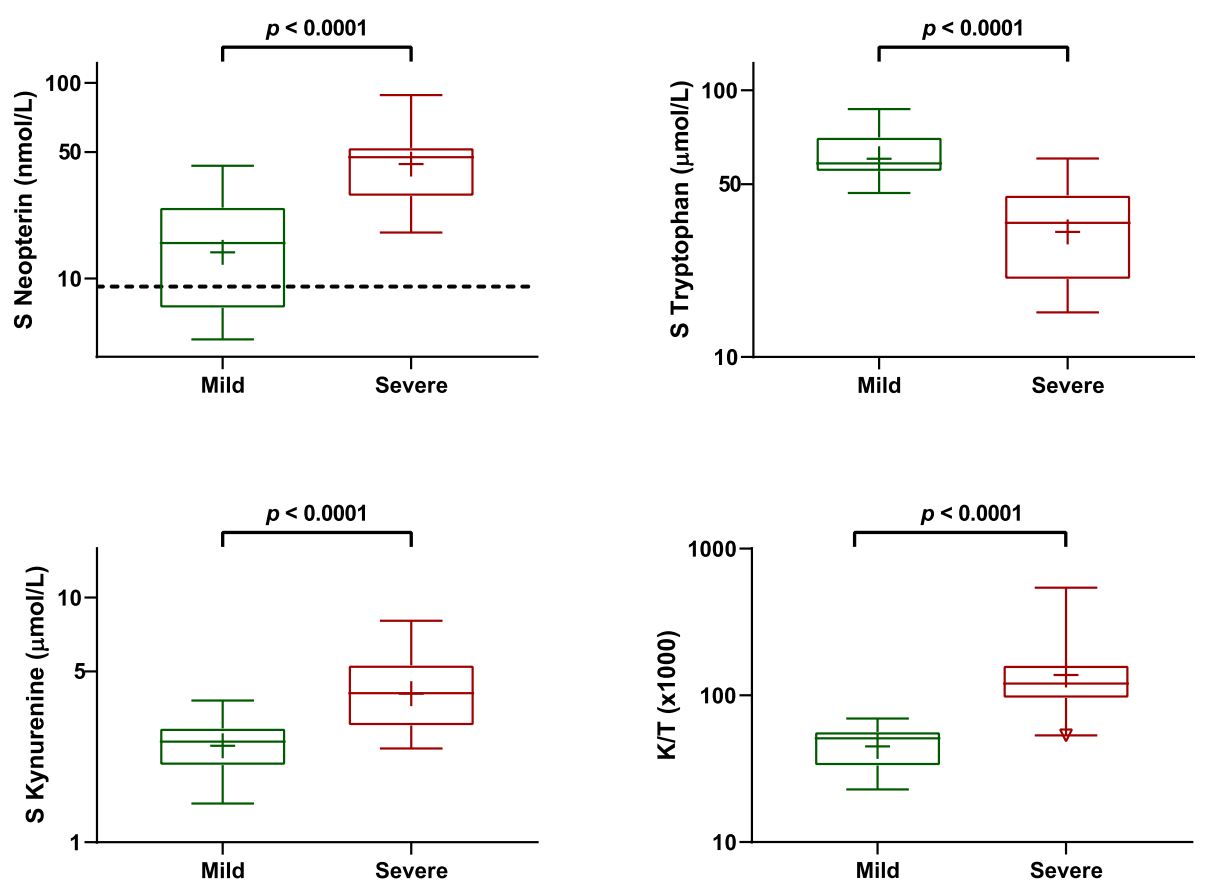

Fig. 1 Concentrations of first measurements of serum neopterin, tryptophan, and kynurenine, as well as kynurenine to tryptophan ratio (K/T) in patients with mild (green) and severe (red) form of COVID-19 $(n=34)$. The dashed line represents the upper normal reference limit of neopterin at $9.1 \mathrm{nmol} / \mathrm{L}$

higher in severe cases compared to mild cases [15]. Besides supporting these findings, our study adds the trajectory of neopterin levels with repeated measurements, where we found that most patients with mild symptoms returned to normal neopterin levels at the end of the study period. In contrast, neopterin levels remained elevated during the course of disease in the severely ill. This probably illustrates a more advanced and prolonged inflammatory state, as suggested by others [16].

The elevated levels of neopterin and IFN-y in severely ill patients with COVID-19 indicate a potent stimulation

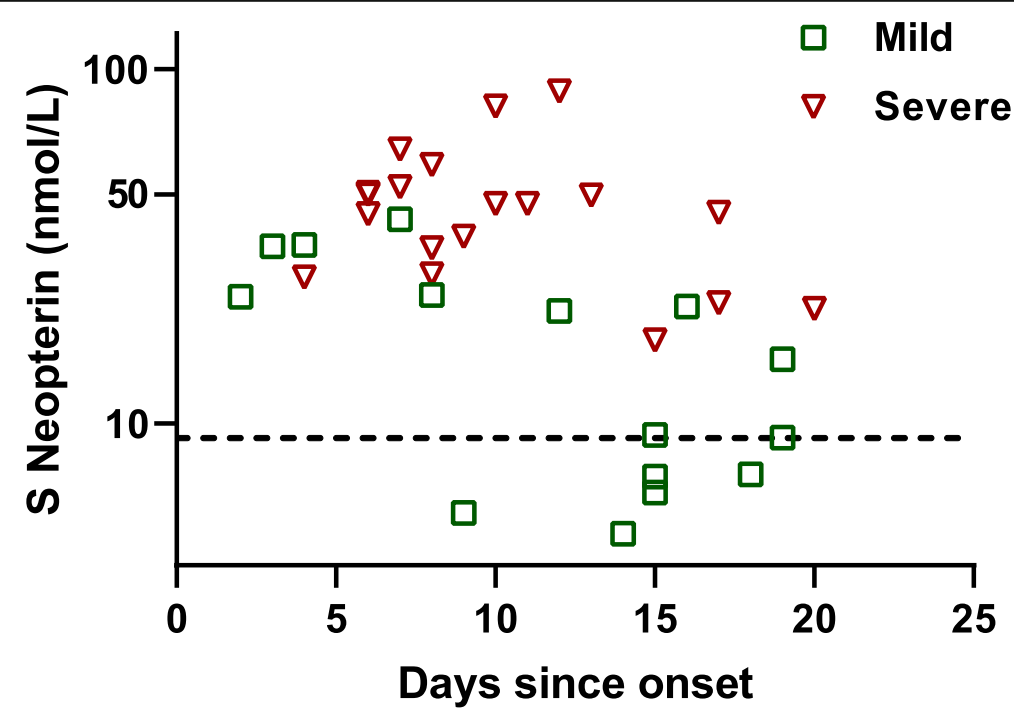

Fig. 2 First measurements of serum neopterin concentrations in patients with mild (green) and severe (red) form of COVID-19 ( $n=34)$. The dashed line represents the upper normal reference limit of neopterin at $9.1 \mathrm{nmol} / \mathrm{L}$. 


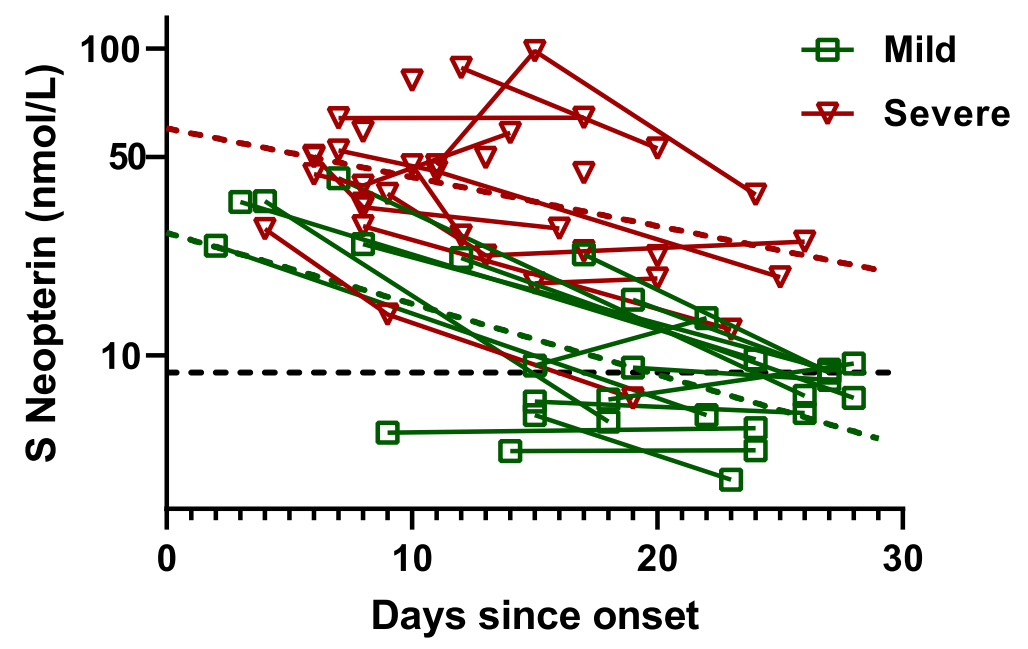

Fig. 3 Repeated measurements of serum neopterin concentrations in patients with mild (green) and severe (red) form of COVID-19 ( $n=34$ ). Severity (mild/severe), days since symptom onset, and age were used as covariates in the linear mixed effect model. The black dashed line represents the upper normal reference limit of neopterin at $9.1 \mathrm{nmol} / \mathrm{L}$.

of the monocyte/macrophage-T cell interplay during the symptomatic period [9]. Our detection of high neopterin concentrations already at day 2 after symptom onset suggests an early inflammatory response to SARSCoV-2. Likewise, the study of SARS patients [3], and also a study of dengue fever [8], found neopterin elevation at the first day of symptoms. The characteristics of neopterin being elevated at an early stage of disease, as well as being associated to disease severity, suggest it as a useful prognostic marker for COVID-19.

The increased KYN to TRP ratio in patients with severe COVID-19 reflects an increased TRP catabolism.
Similar findings have been presented for HIV-infected patients with a progressive disease [17]. TRP deprivation is an effective strategy of the Th1-type immune response to reduce undesirable proliferation of pathogens and infected cells [18], which may be useful for disease control in COVID-19.

When interpreting our results, one must consider the potential involvement of renal function [14]. Acute kidney injury has been found in 50\% of fatal COVID-19 cases [19]. In this context, the lack of creatinine measurements in mild cases make out a limitation of the present study. However, we found no correlation

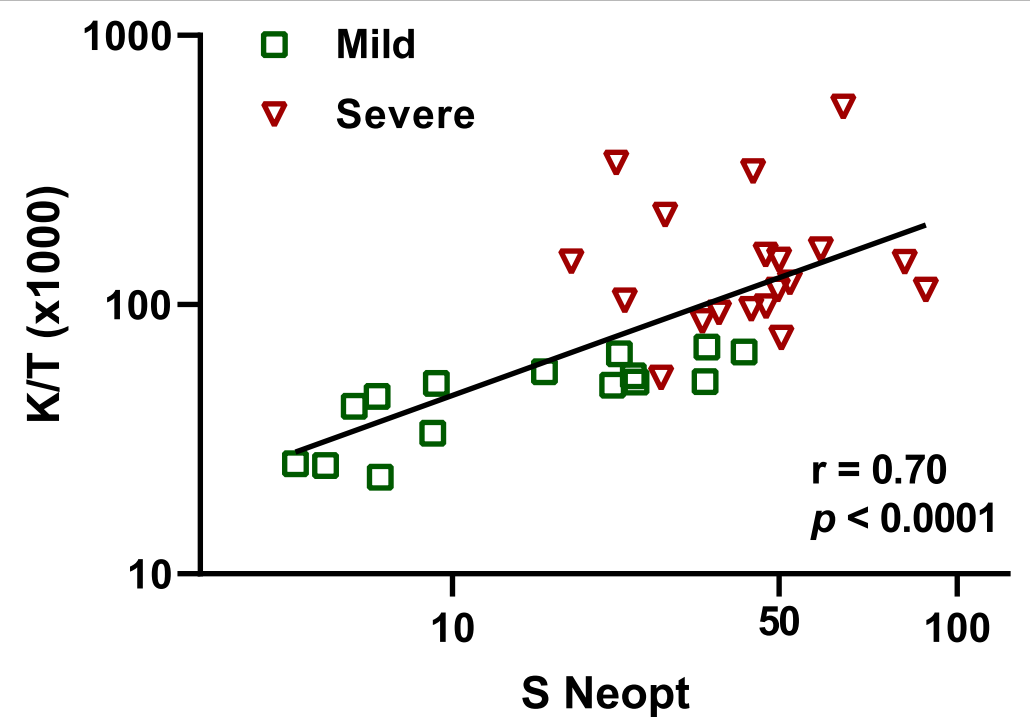

Fig. 4 The correlation between first measurements of serum neopterin ( $\mathrm{S}$ Neopt) concentration and kynurenine to tryptophan ratio (KT) in patients suffering from COVID-19 $(n=34)$ 
between creatinine and neopterin levels in the severe cases at the first measurement.

\section{Conclusions}

The present study shows an association between neopterin levels and severity of COVID-19, and also elevated concentrations early in disease progression. In conclusion, serum neopterin is a potential marker for the prognosis of COVID-19 when detected in blood samples from a few days since symptom onset, but further studies are needed to elucidate how it can be used in the clinic.

\section{Abbreviations}

SARS: Severe acute respiratory syndrome; IFN-y: Interferon-gamma; TRP: Tryptophan; KYN: Kynurenine; IDO: Indoleamine 2,3-dioxygenase; RTPCR: Reverse transcriptase polymerase chain reaction; ELISA: Enzyme-linked immunosorbent assay; CRP: C-reactive protein

\section{Acknowledgements}

Not applicable.

\section{Authors' contributions}

JR and MG were responsible for the conception and design of the study, as well as for acquisition and analysis of data. JMG and DF performed the biochemical analyses. MG, JR, and SN performed the statistical analyses. JR, $M G, J M G, D F, S N$, and LMA took part in drafting the manuscript and approved the final version.

\section{Funding}

This work was supported by the Swedish state, under an agreement between the Swedish government and the county councils (ALF agreement ALFGBG-717531); and by SciLifeLab Sweden (KAW 2020.0182). Open Access funding provided by Gothenburg University Library.

\section{Availability of data and materials}

The datasets used and/or analyzed during the current study are available from the corresponding author on reasonable request.

\section{Ethics approval and consent to participate}

The study has been approved by the Swedish Ethical Review Authority (2020-01771). Participants were enrolled after written informed consent.

\section{Consent for publication}

Not applicable.

\section{Competing interests}

The authors declare that they have no competing interests.

\section{Author details}

${ }^{1}$ Department of Infectious Diseases, Institute of Biomedicine, Sahlgrenska Academy, University of Gothenburg, Gothenburg, Sweden. ²Department of Infectious Diseases, Region Västra Götaland, Sahlgrenska University Hospital, Gothenburg, Sweden. ${ }^{3}$ Institute of Medical Biochemistry, Biocenter, Medical University of Innsbruck, Innsbruck, Austria. ${ }^{4}$ Mathematical Sciences, Chalmers University of Technology, Gothenburg, Sweden. ${ }^{5}$ Institute of Biological Chemistry, Biocenter, Medical University of Innsbruck, Innsbruck, Austria.

Received: 30 July 2020 Accepted: 27 November 2020

Published online: 10 December 2020

\section{References}

1. https://www.who.int/emergencies/diseases/novel-coronavirus-2019.

2. Zunyou W, McGoogan JM. Characteristics of and important lessons from the coronavirus disease 2019 (COVID-19) outbreak in China: summary of a report of 72314 cases from the Chinese Center for Disease Control and Prevention. JAMA. 2020. In press. https://doi.org/10.1001/jama.2020.2648.
3. Zheng B, Cao KY, Chan CPY, et al. Serum neopterin for early assessment of severity of severe acute respiratory syndrome. Clin Immunol. 2005;116:1826. https://doi.org/10.1016/j.clim.2005.03.009.

4. Murr C, Widner B, Wirleitner B, Fuchs D. Neopterin as a marker for immune system activation. Curr Drug Metab. 2002;3:175-87. https://doi.org/10.2174/ 1389200024605082

5. Reibnegger $\mathrm{G}$, Auhuber I, Fuchs D, et al. Urinary neopterin levels in acute viral hepatitis. Hepatology. 1988;8:771-4. https://doi.org/10.1002/hep. 1840080412.

6. Schennach $H$, Hessenberger $G$, Mayersbach P, Schönitzer D, Fuchs D. Acute cytomegalovirus infections in blood donors are indicated by increased serum neopterin concentrations. Med Microbiol Immunol. 2002;191:115-8. https://doi.org/10.1007/s00430-002-0148-8.

7. Zaknun D, Weiss G, Glatzl J, Wachter H, Fuchs D. Neopterin levels during acute rubella in children. Clin Infect Dis. 1993:17:521-2. https://doi.org/10. 1093/clinids/17.3.521.

8. Chan CPY, Choi JWY, Cao KY, et al. Detection of serum neopterin for early assessment of dengue virus infection. J Inf Secur. 2006;53:152-8. https://doi. org/10.1016/j.jinf.2005.11.008

9. Huber C, Batchelor JR, Fuchs D, et al. Immune response-associated production of neopterin. Release from macrophages primarily under control of interferon-gamma. J Exp Med. 1984;160:310-6. https://doi.org/10.1084/ jem.160.1.310.

10. Fuchs D, Möller AA, Reibnegger G, Stöckle E, Werner ER, Wachter H. Decreased serum tryptophan in patients with HIV-1 infection correlates with increased serum neopterin and with neurologic/psychiatric symptoms. J Acquir Immune Defic Syndr. 1990;3:873-6.

11. Schroecksnadel K, Wirleitner B, Winkler C, Fuchs D. Monitoring tryptophan metabolism in chronic immune activation. Clin Chim Acta. 2006:364:82-90 https://doi.org/10.1016/j.cca.2005.06.013.

12. Geisler S, Mayersbach P, Becker K, Schennach H, Fuchs D, Gostner JM. Serum tryptophan, kynurenine, phenylalanine, tyrosine and neopterin concentrations in 100 healthy blood donors. Pteridines. 2015:26:31-6. https://doi.org/10.1515/pterid-2014-0015.

13. Widner B, Werner ER, Schennach H, Wachter H, Fuchs D. Simultaneous measurement of serum tryptophan and kynurenine by HPLC. Clin Chem. 1997:43:2424-6.

14. Fuchs D, Stahl-Hennig C, Gruber A, Murr C, Hunsmann G, Wachter H. Neopterin - its clinical use in urinalysis. Kidney Int Suppl. 1994;47:S8-S11.

15. Ozger HS, Dizbay M, Corbacioglu SK, et al. The prognostic role of neopterin in COVID-19 patients. J Med Virol. 2020:1-6. https://doi.org/10.1002/jmv. 26472.

16. Ye $\mathrm{Q}$, Wang $\mathrm{B}$, Mao J. The pathogenesis and treatment of the 'cytokine storm' in COVID-19. J Inf Secur. 2020;80:607-13. https://doi.org/10.1016/j.jinf. 2020.03.037

17. Huengsberg M, Winer JB, Gompels M, Round R, Ross J, Shahmanesh M. Serum kynurenine-to-tryptophan ratio increases with progressive disease in HIV-infected patients. Clin Chem. 1998:44:858-62.

18. Pfefferkorn ER. Interferon gamma blocks the growth of toxoplasma gondii in human fibroblasts by inducing the host cells to degrade tryptophan. Proc Natl Acad Sci U S A. 1984;81:908-12. https://doi.org/10.1073/pnas.81.3.908.

19. Zhou F, Yu T, Du R, et al. Clinical course and risk factors for mortality of adult inpatients with COVID-19 in Wuhan, China: a retrospective cohort study. Lancet. 2020;395:1054-62. https://doi.org/10.1016/S01406736(20)30566-3.

\section{Publisher's Note}

Springer Nature remains neutral with regard to jurisdictional claims in published maps and institutional affiliations. 